\title{
Is the soleus a sentinel muscle for impaired aerobic capacity in heart failure?
}

Fausto A. Panizzolo ${ }^{1}$, Andrew J. Maiorana ${ }^{2,3}$, Louise H. Naylor ${ }^{1}$, Glen A. Lichtwark ${ }^{4}$, Lawrence Dembo $^{2,5}$, David G. Lloyd ${ }^{6}$, Daniel J. Green ${ }^{1,7}$ and Jonas Rubenson ${ }^{1}$

${ }^{1}$ The School of Sport Science, Exercise and Health, The University of Western Australia, Crawley, WA, 6009, Australia.

${ }^{2}$ Advanced Heart Failure and Cardiac Transplant Service, Royal Perth Hospital, Perth, WA 6000, Australia.

${ }^{3}$ School of Physiotherapy and Exercise Science, Curtin University, Perth, WA 6102, Australia.

${ }^{4}$ The School of Human Movement Studies, The University of Queensland, St Lucia Campus, QLD, Australia

${ }^{5}$ Envision Medical Imaging, Perth, Western Australia, Australia.

${ }^{6}$ Centre for Musculoskeletal Research, Griffith Health Institute, Griffith University, Gold Coast, QLD, 4222, Australia.

${ }^{7}$ Research Institute for Sport and Exercise Science, Liverpool John Moores University, Liverpool, United Kingdom.

Running title: skeletal muscle and aerobic capacity in CHF

Author for correspondence:

Dr. Jonas Rubenson

The School of Sport Science, Exercise and Health, The University of Western Australia, Crawley, WA, 6009, Australia, Tel: + 6186488 5533, Email: jonas.rubenson@uwa.edu.au

\section{DISCLOSURES}

No conflicts of interest, financial or otherwise, are declared by the authors. This work was supported by a Grant-in-Aid (G09P 4469) from the National Heart Foundation of Australia to J.R., D.J.G., A.J.M. and D.G.L., and a thesis dissertation grant from the International Society of Biomechanics to F.A.P. 


\section{ABSTRACT}

Purpose: Skeletal muscle wasting is well documented in chronic heart failure (CHF). This paper provides a more detailed understanding of the morphology behind this muscle wasting and the relationship between muscle morphology, strength and exercise capacity in CHF. We investigated the impact of CHF on leg lean mass, detailed muscle-tendon architecture of the individual triceps surae muscles (soleus, SOL; medial gastrocnemius, MG and lateral gastrocnemius, LG) and how these parameters relate to exercise capacity and strength. Methods: 11 CHF patients and 15 agematched controls were recruited. Leg lean mass was assessed by dual energy X-ray absorptiometry (DXA) and the architecture of skeletal muscle and tendon properties by ultrasound. Plantarflexor strength was assessed by dynamometery. Results: Patients with CHF exhibited 25\% lower combined triceps surae volume and physiological cross-sectional area (PCSA) compared to control subjects $(\mathrm{p}<0.05)$, driven in large part by reductions in the SOL. Only the SOL volume and the SOL and MG PCSA were statistically different between groups after normalizing to lean body mass and body surface area, respectively. Total lower leg lean mass did not differ between CHF and control subjects, further highlighting the SOL specificity of muscle wasting in CHF. Moreover, the volume of the SOL and plantarflexor strength correlated strongly with peak oxygen uptake ( $\dot{V}_{2}$ peak ) in patients with CHF. Conclusions: These findings suggest that the SOL may be a sentinel skeletal muscle in CHF and provide a rationale for including plantarflexor muscle training in CHF care.

Keywords: CHRONIC HEART FAILURE, SKELETAL MUSCLE, SOLEUS, EXERCISE CAPACITY 


\section{INTRODUCTION}

\section{Paragraph number 1}

Impaired exercise capacity is a hallmark symptom of chronic heart failure (CHF) (8, 9). The important contribution of peripheral, as against cardiac, factors in the functional limitation which characterizes CHF has long been recognized and is reinforced by the finding that cardiac function is poorly correlated with exercise capacity (9). Moreover, it appears that dysfunctional skeletal muscles have a direct influence on the reduced exercise capacity evident in patients with CHF (8), and may contribute to a progressive deterioration in clinical status. These findings resulted in the 'muscle hypothesis' of CHF (8), which proposes that skeletal muscle dysfunction is a key factor responsible for reduced exercise capacity in this group.

\section{Paragraph number 2}

Consistent with the 'muscle hypothesis', muscle wasting is common in patients with CHF (8, 24), especially in advanced disease. It has been found that limb muscle mass is correlated with reduced exercise capacity (peak oxygen consumption; $\dot{V} o_{2}$ peak) (7). Indeed, a recent analysis of 200 CHF patients (12) revealed that reduced muscle mass is independently associated with lower absolute $\dot{V} o_{2}$ peak $\left(\mathrm{ml} \mathrm{min}^{-1}\right)$ when age, sex, ejection fraction and co-morbidities are accounted for, and that muscle mass is directly linked to disease progression.

\section{Paragraph number 3}

Skeletal muscle wasting is therefore highly relevant to the progression and treatment of CHF. However, whilst an overall reduction in skeletal muscle mass in CHF is evident, a more detailed understanding of the morphology behind muscle wasting and the relationship between muscle morphology, muscle functional capacity (strength) and exercise capacity in CHF remains unexplored. For example, it is not known whether loss of mass occurs evenly across lower limb 
muscles, or if certain muscles exhibit proportionately more/less muscle wasting. It is conceivable that predominantly slow-twitch muscles may be more markedly affected, given the reported increase in the proportion of type-II fibers (8), the reduced blood flow and vascular transport capacity in CHF (31) and reports that type-I fiber atrophy is related to severity of the disease (10). It is also possible that muscle wasting is more prominent in the distal leg muscles, given their greater functional role in generating the mechanical work and power for walking and for the maintenance of posture (25). The "architectural” changes that underlie the loss of muscle mass are also poorly understood. Loss of muscle mass may occur through decreased fiber length, with physiological cross-sectional area (PCSA) of the muscle remaining unaffected, or through loss of PCSA with fiber length remaining unaffected, or a combination of these. The distinction is important, given that whole-muscle architecture is a strong predictor of skeletal muscle mechanical capacity (e.g. strength) (21) and may also impact muscle energetics (35). Finally, the mechanical function of a muscle is also dependent on in-series tendon properties. Tendons function by storing and releasing elastic energy during locomotion, thereby influencing the contractile behavior of muscle, including work and power production and efficiency (20). Tendon mechanics are known to be closely associated with muscle wasting in ageing and chronic unloading $(32,38)$. To the best of our knowledge, a detailed structural analysis combining muscle volume, fiber length and pennation angle along with tendon properties has not previously been undertaken in human CHF, nor have they been analysed in relation to muscle strength or exercise capacity in this group.

\section{Paragraph number 4}

The aims of the present study were therefore: 1) To assess whether muscle wasting in CHF is constant across different muscles of the lower limb, in particular across synergist muscles known to differ in fiber composition and function; 2) To assess the detailed architecture of synergist skeletal muscle and tendon properties in patients with CHF, compared to healthy age-matched control participants (with similar adiposity and exercise activity); 3) To assess how skeletal muscle 
architecture, muscle strength and exercise capacity are related among CHF patients and control participants. To address these questions we analyzed leg lean mass and performed a detailed architectural assessment of the triceps surae (calf muscles) and Achilles tendon, along with functional strength measurements of this muscle group. The triceps surae [soleus (SOL), medial gastrocnemius (MG) and lateral gastrocnemius (LG)] were chosen because they offer a highly relevant comparison between synergist muscles known to possess different fiber types (predominantly slow twitch in SOL vs. mixed fibers in gastrocnemius) (13). Furthermore, the triceps surae muscles are functionally important, representing the main source of power during walking (25) and have been shown to be a major locus of gait impairment in ageing $(11,34)$.

\section{MATERIALS AND METHODS}

\section{Paragraph number 5}

Subjects. We recruited 11 subjects with CHF (7 men, 4 women; NYHA II-IV; EF = $30 \pm 10$ \%; age: $61.8 \pm 10.0$ yr; height: $1.68 \pm 0.10 \mathrm{~m}$; weight: $72.8 \pm 18.0 \mathrm{~kg}$ ). Criteria for exclusion included severe renal (creatinine $>250 \mathrm{mmol} / \mathrm{L}$ or eGFR $<30 \mathrm{ml} / \mathrm{min} / 1.73 \mathrm{~m} 2$ ) or hepatic (bilirubin $>50 \mathrm{mmol} / \mathrm{L}$ ) dysfunction, unexplained anaemia (haemoglobin $<100 \mathrm{~g} / \mathrm{L}$ ) or thrombocytopaenia (platelets $<100 x 109 / \mathrm{L}$ ), unstable angina or exercise-induced ischaemia at low exercise levels, severe aortic stenosis, severe mitral or aortic regurgitation, or hypertrophic cardiomyopathy. All CHF subjects were medically stable at the time of testing. The CHF group was attending supervised exercise training 2-3 times per week for $\sim 1$ hour per session (treadmill walking, cycle ergometry and resistance training) as part of their multidisciplinary care.

The control group consisted of 15 healthy subjects recruited from the local community ( 9 men, 6 women; age: $60.7 \pm 6.2 \mathrm{yr}$; height: $1.72 \pm 0.07 \mathrm{~m}$; weight: $69.9 \pm 8.6 \mathrm{~kg})$. Eleven of these control subjects were recruited because they matched the CHF group for age, sex and adiposity and had similar levels of exercise to those of the CHF patients, and were used in comparative statistics. The 
additional 4 controls were recruited specifically to validate the ultrasound measurement of muscle volume (a total of 6 participants were used for ultrasound validation). All subjects provided written informed consent prior to participating in the study. All procedures were approved by the Human Research Ethics Committee at The University of Western Australia (approval ID: RA/4/1/2533) and Royal Perth Hospital (approval ID: 2011/019).

\section{Paragraph number 6}

Body composition measurements. Overall body composition was determined using dual energy X-ray absorptiometry (DXA) (Luna Prodigy, encore 2004, GE Medical Systems, Madison, WI, USA) on each subject including a measurement of total fat-free mass, total fat mass and bone mineral density. Lean mass for the lower limbs was computed separately by selecting a region of interest from the greater trochanter to the pubic symphysis and included the leg and foot.

\section{Paragraph number 7}

Triceps surae volume calculation. Due to the presence of internal cardioverter defibrillators in 10 of the 11 CHF subjects, rendering MRI unsuitable, muscle volume was computed using a three-dimensional ultrasound technique (3DUS) based on a combination of B-mode ultrasound imaging and 3D motion data (2). Subjects kneeled with their right leg in a custom-designed plastic water bath equipped with an adjustable metal footplate designed to secure the subject's foot and to set the ankle joint angle to $0^{\circ}$ (i.e. foot perpendicular to the tibia), with the knee in $10^{\circ}$ flexion (near maximal extension). The water bath was maintained within a temperature range of $24-25^{\circ} \mathrm{C}$ to ensure that temperature-dependent variations in the speed of sound were minimized.

\section{Paragraph number 8}

Ultrasound images (Telemed, Echoblaster128, Lithuania; 7.5 MHz linear array probe) and 3D marker trajectories of a probe-mounted marker cluster that defined the probe position in space (5- 
camera Vicon MX system, $250 \mathrm{~Hz}$; Oxford Metrics, UK) were used to calculate muscle volume. The ultrasound and motion capture systems were synchronized using a $3 \mathrm{~V}$ square pulse generated at the beginning of the images collection by the ultrasound device. The subjects' lower leg was scanned longitudinally from the popliteal cave to the calcaneus. Three sweeps were performed to scan the whole triceps surae, starting at the lateral side and ending medially so that each sweep overlapped by a minimum of $20 \mathrm{~mm}$. A customized MATLAB script (The MathWorks Inc., USA) was then used to combine ultrasound images and 3D position data and to convert them to Stradwin format (Medical Imaging Research Group, Cambridge University Engineering Department, UK) (2). Manual segmentation of the muscles was performed in Stradwin software by a single investigator (FAP) on approximately 30 slices covering the length of the muscle group (inter slice gap $15 \mathrm{~mm}$ ). Muscle volumes for the three muscles under investigation (SOL, MG and LG) were computed. The segmentation procedure and analysis of muscle volumes was conducted in a blinded manner to avoid operator bias. To account for the possible variability during data collection and manual segmentation, the collection procedure described above was repeated three times for each subject and an average volume for the SOL, MG and LG computed.

\section{Paragraph number 9}

We conducted comparisons between our ultrasound volume measurements and those obtained from axial plane MRI scans of the lower leg in a subsample $(n=6)$ of control subjects who were able to undergo this form of imaging. MRI scans were collected using a Magnetom Espree 1.5T scanner (Siemens, Erlangen, Germany) with the subjects' leg position matched to that of the 3DUS experiment (see Text, Supplemental Digital Content 1, which describes the full details of the MRI procedures). The accuracy of the CSA of the Achilles tendon was also assessed by comparing ultrasound and MRI-derived images. 


\section{Paragraph number 10}

Fascicle length, pennation angle and muscle physiological cross-sectional area (PCSA). The ultrasound probe described above was first placed over the middle of the selected muscle belly (SOL, MG, LG) following the guidelines of Rubenson et al. (36), with its longitudinal axis aligned with the orientation of the fascicle. Fascicle length and pennation angle at neutral ankle $\left(0^{\circ}\right)$ and $10^{\circ}$ knee flexion joint angles (approximating the angle where passive fascicle forces and net joint torque approach zero, Ref. 36, 1) were calculated in ImageJ, as per Rubenson et al. (36). For a better characterization of the muscle anatomy, fascicle lengths of the SOL and MG muscles were also calculated in the proximal, medial and distal locations of the muscle, with approximately $4 \mathrm{~cm}$ spacing between each location.

\section{Paragraph number 11}

The functional range of mid-belly fascicle length in the SOL and MG were also assessed using a dynamometer (Biodex, M3, Shirley, NY, USA) with continuous recording of passive muscle fascicle lengths and joint angle across the ankle range of motion (the most dorsiflexed and most plantarflexed position tolerated by the participant using a passive isokinetic ( $5 \% / \mathrm{sec})$ protocol). To assess the length range of the SOL the knee was positioned at $120^{\circ}$ of flexion, minimizing the passive force contribution of the MG and LG (1). The length range of the MG was assessed with the knee extended. Ankle joint angles were obtained using a 3-camera OptiTrack motion capture system (Natural Point, Corvallis, OR; $100 \mathrm{~Hz}$ ). The assessment of regional muscle lengths and functional length range was performed only on the MG and SOL in order to shorten the testing time and avoid discomfort of prolonged experimentation in the CHF group. For each muscle, physiological cross-sectional area (PCSA) was calculated as:

$$
\text { PCSA }=(\text { muscle volume } * \cos (\theta)) / \text { fascicle length }
$$

Fascicle length and pennation angles used for the PCSA calculation were obtained at the mid-belly position of the muscle from images collected in the 3DUS water bath experiment. PCSA was 
expressed in $\mathrm{cm}^{-2}$ and subsequently normalized to body surface area (BSA) calculated as per Moesteller (27).

\section{Paragraph number 12}

Tendon length and cross-sectional area. A scaled subject specific musculoskeletal model in OpenSim 2.0.2 (1) was used to estimate the muscle-tendon unit lengths for each subject at $0^{\circ}$ of plantarflexion and $10^{\circ}$ knee flexion. Tendon length, defined as the free tendon plus aponeurosis (series elastic element), was subsequently calculated as the difference between the estimated muscle-tendon unit length of each of the SOL, MG and LG obtained by the scaled OpenSim model and the estimated muscle length from ultrasound of the respective muscles (fascicle length $* \cos (\theta)$ ) at an ankle angle of $0^{\circ}$ (Fig.1). An average of the three tendon lengths was obtained with this procedure (see Text, Supplemental Digital Content 2, which describes the triceps surae tendon length estimates).

\section{Paragraph number 13}

Using the same setup and settings described for the determination of 3D muscle volume, ultrasound images of Achilles tendon cross sectional area (CSA) were taken at the level of the medial malleolus to standardize the measurements across participants. To obtain tendon CSA, images were manually digitized in ImageJ.

\section{Paragraph number 14}

Strength. Strength measurements were assessed by means of a robotic dynamometer (M3, Biodex, Shirley, NY, USA). Subjects sat with their right foot strapped to a custom built foot plate designed to align the centre of rotation of the ankle with that of the Biodex and to minimize heel movement during the contraction. Subjects performed three maximum voluntary contractions with the knee extended ( $10^{\circ}$ flexion) and the ankle dorsiflexed to $10^{\circ}$, approximating the angle at which 
the maximum torque is produced at the ankle joint $(1,36)$. A minimum of two minutes was observed between repetitions. Peak torque was calculated as the difference between the maximum torque during contraction and the torque measured at rest arising primarily from the weight of the rig and foot and small passive muscle forces (36). Analog data were collected using a CED data acquisition system running Spike2 V7 software (Micro1401-3; Cambridge Electronic Design, Cambridge, UK; $2000 \mathrm{~Hz}$ ) and processed by means of a custom written program in MATLAB (The Mathworks, Natick, MA). For each subject the trial with the highest peak torque was reported and used for comparisons.

\section{Paragraph number 15}

Exercise capacity. Peak oxygen uptake $\left(\mathrm{V}_{2}\right.$ peak $)$ was assessed using a purpose-designed incremental walking protocol on a motorized treadmill, starting at a speed $20 \%$ slower than each individual's preferred speed with stepwise increases in speed and grade every 3 min until the participant reached volitional exhaustion. Indirect calorimetry was conducted using a Vmax Encore gas analysis system (Sensormedics, Yorba Linda, California), which enabled the measurement of

expired gas concentrations and volumes. Absolute $\dot{V} o_{2}$ peak was expressed in $\mathrm{ml} \mathrm{min}^{-1}$ and normalized to body mass $\left(\mathrm{ml} \mathrm{kg}^{-1} \mathrm{~min}^{-1}\right)$. All tests were performed by an exercise physiologist (AJM) experienced with undertaking cardiopulmonary exercise testing in patients with heart failure (22).

\section{Paragraph number 16}

Statistics. Differences in muscle volume (absolute and lean body mass-normalized) and PCSA (absolute and BSA-normalized) were analyzed using a two-way between groups ANOVA with group (CHF/control) and muscle synergist (SOL/MG/LG) as fixed factors. Differences in muscle fascicle length were analyzed using a two-way between groups ANOVA with group (CHF/control) 
and muscle synergist (SOL/MG/LG) as fixed factors for the mid-belly location. A two-way between groups ANOVA with group (CHF/control) and muscle synergist (SOL/MG) as fixed factors was performed for the proximal and distal locations. If a significant interaction effect between group and muscle was observed $(\mathrm{p}<0.05)$ a one-way ANOVA with group as a fixed factor was performed for each muscle. A one-way ANOVA was used to determine differences in the fascicle length range and pennation angle, leg lean mass, strength and tendon properties between groups. ANOVAs were performed in SPSS using a Bonferroni post hoc analysis (IBM, Statistic 21, USA).

\section{Paragraph number 17}

Linear regression was used to determine correlations between $\dot{V} o_{2}$ peak and a) lean leg mass, b) muscle volume, c) muscle PCSA (total triceps surae as well as single muscles) and d) strength, and between the muscle PCSA and tendon cross-section. A correlation analysis was also performed between 3DUS- and MRI-derived measurements of muscle volume and the agreement between the two techniques was assessed using the limits of agreement method (6). Correlation coefficients (r) and significance level $(\mathrm{p}<0.05)$ were determined in SPSS. Values presented are mean \pm SD unless otherwise stated.

\section{RESULTS}

\section{Paragraph number 18}

Subject characteristics. No significant differences in age, height or mass or other anthropometric characteristics were observed between control and CHF groups; leg length was 0.38 $\pm 0.04 \mathrm{~m}$ in the CHF group and $0.40 \pm 0.02 \mathrm{~m}$ in the control group ( $\mathrm{p}=0.14$ ); adiposity (fat body mass) was $21.3 \pm 11.3 \mathrm{~kg}$ in CHF group and $17.8 \pm 7.2 \mathrm{~kg}$ in the control group (p=0.38); $\dot{V} o_{2}$ peak was $15.5 \pm 3.0 \mathrm{ml} \mathrm{kg}^{-1} \mathrm{~min}^{-1}$ in CHF and $35.6 \pm 8.3 \mathrm{ml} \mathrm{kg}^{-1} \mathrm{~min}^{-1}$ in control $(\mathrm{p}<0.0001)$. It should 
be noted that one CHF patient and two control participants did not undergo strength testing due to discomfort and in one CHF subject image degradation prevented an accurate SOL volume.

\section{Paragraph number 19}

DXA lean mass and triceps surae muscle volume and PCSA. Total body lean mass (49.0 \pm $10.6 \mathrm{~kg}, \mathrm{CHF} ; 49.2 \pm 8.3 \mathrm{~kg}$, control; $\mathrm{p}=0.95)$ and leg lean mass (7.6 $\pm 1.9 \mathrm{~kg}, \mathrm{CHF} ; 8.3 \pm 1.5 \mathrm{~kg}$, control; $\mathrm{p}=0.4$ ) as assessed by DXA did not significantly differ between groups. The two-way ANOVA determined a main effect of both group and muscle on absolute volume $(p=0.015$ and $p<$ 0.0001, respectively) and volume normalized to lean body mass ( $\mathrm{p}=0.007$ and $\mathrm{p}<0.0001$, respectively). Post hoc analysis found that volumes collapsed over groups were statistically different between all muscles $(\mathrm{p}<0.01)$. The combined muscle volume of the triceps surae was, on average, 27.2\% higher in the control group compared to the CHF group (632 $\pm 169 \mathrm{ml}$ vs $497 \pm 155$ ml; Fig. 2a). A significant interaction effect between group and muscle was found in the lean massnormalized volume $(\mathrm{p}=0.03)$. Post hoc analysis revealed a significant difference in the normalized SOL muscle volume between the CHF and control group (20.5\% smaller) but not in the MG or LG (Fig. 2b). A trend towards an interaction effect was also observed between group and muscle in the absolute muscle volume $(\mathrm{p}=0.08)$ and was largely due to the small difference in LG volume between the CHF and control group (8.7\% smaller) compared to the SOL (27.5\% smaller) and the MG (20.9\% smaller)

\section{Paragraph number 20}

From the volume renderings of the triceps surae muscle group (Fig. 3a), an average difference of $20 \pm 46 \mathrm{ml}$ was observed between 3DUS and MRI techniques for the combined triceps surae muscles. This difference amounted to $2.5 \pm 6.3 \%$ difference between measurements of the total triceps surae volume between the two techniques. The average difference in volume for each 
individual muscle was $4.2 \%$. The overall correlation coefficient between 3D Ultrasound and MRI was 0.989 (Fig. 3b) and the 95\% confidence interval ranged between +47 and $-33 \mathrm{ml}$ (Fig. 3c).

\section{Paragraph number 21}

No main effect of group on fascicle length or interaction effect between group and muscle were observed in any ANOVAs. However, a main effect of muscle on fascicle length was observed for the comparison in the distal muscle location $(\mathrm{p}=0.04$; Table 1$)$. No significant differences were found in fascicle lengths or joint angles across the functional ankle range of motion between the CHF and control subjects. A main effect of both group and muscle were found for absolute PCSA ( $p<0.0001$ for both). A main effect of both group and muscle were also found for BSA-normalized PCSA (p $<0.0001$ for both). Post hoc analysis found that PCSA collapsed across groups were statistically different between all muscles $(\mathrm{p}<0.01)$. The PCSA of triceps surae in the control group was, on average, $23.7 \%$ higher than in the CHF group (Fig. 2c). Interaction effects between muscle and group were found in the absolute PCSA $(p=0.042)$ and in the BSA-normalized PCSA $(p=$ 0.024). Post hoc analysis found significant differences between the SOL and MG absolute PCSA from the CHF and control groups but not for the LG. The average absolute PCSA of the SOL and MG were 22.2\% and 25.3\% smaller in the CHF group than in the control group, respectively (Fig. 2c). Similar results were observed for the BSA-normalized PCSA whereby only the SOL and MG were significantly different between groups (Fig. 2d).

\section{Paragraph number 22}

A significant correlation between total lean lower limb mass and absolute $\dot{V} o_{2}$ peak (ml min ${ }^{-1}$; r $=0.82, \mathrm{p}=0.004)$ and total triceps surae volume and absolute $\dot{V} o_{2}$ peak $\left(\mathrm{ml} \mathrm{min}^{-1} ; \mathrm{r}=0.93, \mathrm{p}<\right.$ 0.0001) was observed in the CHF group (Fig. 4?). When analyzed on an individual muscle level, only the SOL body-mass normalized volume was found to correlate with the body mass normalized 
$\dot{V} o_{2}$ peak (ml kg-1 $\mathrm{min}^{-1}$ ) in the CHF group ( $\mathrm{r}=0.72, \mathrm{p}=0.018$; Fig.4?). The control group exhibited weaker correlations between lean leg mass and absolute $\dot{V} o_{2}$ peak and triceps surae volume and absolute $\dot{V} o_{2}$ peak $(r=0.63, \mathrm{p}=0.06$ and $\mathrm{r}=0.58, \mathrm{p}=0.08$, respectively). Unlike the CHF group, body mass normalized data were not correlated in the control group. Muscle PCSA was independent of $\dot{V} \mathrm{O}_{2}$ peak in all muscles in both the CHF and control groups.

\section{Paragraph number 23}

Tendon architectural parameters. The cross-sectional area of the Achilles tendon was significantly smaller in CHF patients compared to the control group (59.2 $\pm 16.1 \mathrm{~mm}^{-2}$ vs. $73.4 \pm$ $20.0 \mathrm{~mm}^{-2}$ respectively, $\mathrm{p}=0.046$ ). However the estimate of tendon length was not different between the CHF $(27.4 \pm 2.7 \mathrm{~cm})$ and control $(28.6 \pm 2.3 \mathrm{~cm})$ groups $(\mathrm{p}=0.2)$. A trend towards a positive correlation between tendon CSA and the triceps surae PCSA existed for CHF $(r=0.65, \mathrm{p}=$ 0.06) but not for the control group $(r=0.35, \mathrm{p}=0.3)$.

\section{Paragraph number 24}

Strength measurements. Body-mass normalized peak torque $\left(\mathrm{N} \mathrm{m} \mathrm{kg}^{-1}\right)$ was $13.3 \%$ greater in the control group vs. the CHF group, although this difference was not statistically different $(\mathrm{p}=0.3$; Fig. 5a.). Peak torque normalized by the triceps surae PCSA $\left(\mathrm{N} \mathrm{m} \mathrm{cm}^{-2}\right)$ was $3.6 \%$ smaller (nonsignificant) in the control group vs. the CHF group ( $p=0.8$; Fig. 5b). A strong correlation between body-mass normalized peak torque and $\dot{V} o_{2}$ peak was found in the CHF group $(\mathrm{r}=0.75 ; \mathrm{p}<0.01)$ but there was no such relationship in the control group $(r=0.25 ; p=0.5$; Fig. $5 c)$. 


\section{DISCUSSION}

\section{Paragraph number 25}

There is growing evidence that skeletal muscle is adversely affected in patients with CHF and that it contributes to the reduced exercise capacity characteristic of this condition. Skeletal muscle wasting, in particular, has been shown to be strongly correlated with exercise capacity and disease progression in CHF (12), yet little data exists on the specifics of wasting between muscles, or the involvement of whole muscle-tendon morphology in muscle wasting. The present study investigated total leg lean mass and detailed muscle-tendon architecture and strength of the triceps surae muscles (calf muscles), an important functional muscle group for maintaining posture and for locomotion (25). We assessed architectural and strength differences between CHF and control subjects, as well as relationships with exercise capacity. Whilst there were no detectable differences in lower limb lean mass as measured by DXA, a clear reduction in the size of the SOL and to a lesser extent the MG, and a reduction in Achilles tendon CSA, were observed in the CHF compared with control subjects. However, these differences did not translate to statistically significant differences in strength. Furthermore, SOL muscle volume and plantarflexor strength were found to strongly correlate with exercise capacity in the CHF group, but not in the control group. These findings offer the possibility that the distal lower limb muscles, and the SOL in particular, may be key skeletal muscles determining exercise capacity and function in CHF patients.

\section{Paragraph number 26}

Is muscle wasting in CHF muscle-specific? Whilst several studies have identified generalized muscle wasting in CHF (12, 24), little is known about the specificity of muscle loss. Interestingly, our 3DUS data revealed that muscle wasting was not uniform across the three muscles of the triceps surae. Although the overall reductions in triceps surae muscle volume and PCSA observed in this study are comparable to one previous MRI-based report assessing triceps surae volume in CHF (24), our novel observation is that the reductions in these parameters are seen only in the SOL, and 
(with respect to volume) to a somewhat lesser extent in the MG. The lack of atrophy in the LG might be related to a difference in muscle mechanical function (16), or possibly a consequence of its relatively small size and limited contribution to posture (16). The more pronounced reduction in muscle volume in the SOL compared to the MG in CHF patients (Fig. 2; in particular when normalized to total leg lean mass) could suggest that muscle wasting is sensitive to muscle aerobic capacity and fiber composition, given the known differences in type I vs. type II fiber distribution between these muscles (13). Greater CHF-induced atrophy in type I compared to type II fibers has been reported for the human vastus lateralis (40), although the opposite has been found in other work reporting fiber type-specific fiber atrophy in CHF (23). In a rat model of CHF, type I fiber atrophy has been correlated to the severity of the disease (10) and, recently, significant atrophy in the rat soleus (predominantly type I fibers) but not the plantaris has been observed (29). Alternatively, the SOL volume may be more affected in CHF due to reduced blood flow (31), or possibly because the SOL has been shown to provide the majority of mechanical work among the lower limb muscles during walking (25) and thus the effect of CHF may be augmented in this functionally important muscle.

\section{Paragraph number 27}

Whereas ultrasound-based measurements of triceps surae volume revealed marked reduction in muscle size ( 30\%), DXA measurements of total lower limb lean mass were, surprisingly, only slightly lower (non-significant) in CHF patients compared to the control group. The $~ 0.7 \mathrm{~kg}$ (7.8\%) reduction in total lower limb lean mass is similar to that reported by Toth et al. (39), who also found non-significant differences in lean mass between CHF and an age-matched control group. When taken together, the high-fidelity 3DUS of the calf muscles and whole-limb DXA recording suggest that the SOL and MG may be particularly prominent, and possibly early sites of muscle loss in CHF. Fülster et al. (12) found that 20\% of CHF patients (NYHA class II-III) exhibited muscle wasting compared with a healthy reference group. Using their criteria, only $30 \%$ of participants in 
the current study would be diagnosed with generalized leg muscle wasting, yet these patients exhibited muscle wasting in the SOL and MG. It seems likely that muscle-specific changes in the triceps-surae may have gone undetected in previous analyses of overall leg lean mass using DXA. This may relate to the relatively small size of the triceps surae muscles ( $\sim 500 \mathrm{~g})$ compared to the total leg lean mass ( $7.5 \mathrm{~kg})$ and the lower sensitivity of DXA ( 300g sensitivity in detecting muscle mass; Ref. 19) compared to 3DUS ( 35 ml sensitivity; Fig. 3c).

\section{Paragraph number 28}

We are unaware of previous studies describing muscle-specific atrophy in CHF. Our data offer the intriguing possibility that calf muscle volume, and the SOL in particular, may be a sentinel muscle for early muscle wasting in this disease. Greater distal muscle atrophy has previously been documented in ageing (27) and in other conditions of unloading, such as bed rest (18, 32) and space flight (32). However, in these studies a more prominent reduction in total lower limb lean mass was observed compared to the present study. We propose that a distal-to-proximal gradation in muscle loss may occur due to the prominent role of the distal muscles in body support and propulsion (25). Muscle wasting may be amplified, especially in the major plantarflexor muscles compared to the other leg muscles, including the LG (16), that have a lower contribution to these functions in activities of daily living, and in particular walking. The SOL and MG play a key role in supporting the trunk during single-leg stance and pre-swing (33) and the SOL is fundamental for the horizontal acceleration in the late stance phase, which is necessary to provide propulsion to the trunk (33). Because of their importance to gait mechanics, it can be argued that a $\sim 25 \%$ reduction in normalized muscle volume and PCSA in the SOL will have a negative impact on function. It is reasonable to hypothesize, therefore, that alterations in gait and movement mechanics and fatigue in CHF will result as a consequence of the reduction in size of the triceps surae, and especially the SOL. 


\section{Paragraph number 29}

The relationship between muscle-tendon architecture and wasting in chronic heart

failure. Muscle architecture analyses revealed that the loss of muscle volume is primarily a result of a loss of muscle PCSA, with muscle length and pennation angles remaining relatively unchanged. Age-related muscle wasting, on the other hand, has been reported to result, in part, from a reduction in muscle length (38), although recent work suggests that the active muscle lengths in the MG may be unaffected by age (3). Our findings therefore suggest a pattern of CHF muscle atrophy that is distinct from that associated with aging per se. Given that volume (17) and PCSA (21) influence a muscle’s mechanical work and force capacity, respectively, our findings suggest a biomechanical basis for impaired function of the calf muscles in CHF patients. It was somewhat surprising, therefore, that a more pronounced reduction in strength was not observed between the $\mathrm{CHF}$ and control groups in the present study. Interestingly, a lack of statistically significant differences in ankle strength is consistent with previous measurements of voluntary plantarflexor torque in CHF (14), and possibly reflect variability in voluntary torque measurements arising from factors such as neural drive, co-contraction, muscle moment arm lengths (4) and/or fiber operating lengths (36). Despite the lack of statistical differences in absolute strength, it is also worth noting that the differences between groups are minimized when normalized for PCSA.

\section{Paragraph number 30}

Similar to other studies addressing triceps surae muscle loss (18), the current study also showed that the Achilles tendon undergoes a concurrent reduction in cross-sectional area in CHF patients. Tendon remodeling occurs in response to the mechanical loading stimulus (37) and it follows, therefore, that the reduced triceps surae muscle size in the CHF patients may be associated with reduced habitual in vivo muscle forces. 


\section{Paragraph number 31}

The relationship between muscle architecture, strength and exercise capacity in chronic heart failure. Previous studies have reported significant correlations between estimates of skeletal muscle mass and functional capacity in $\operatorname{CHF}(12,24)$. Indeed, several studies have reported correlations between total lower limb lean mass or the size of specific muscle groups and $\dot{V} o_{2}$ peak (typically expressed as absolute $\dot{V} o_{2}$ peak $\mathrm{ml} \mathrm{min}^{-1}$ ). Our findings suggest, for the first time, that a principal reason for such leg muscle mass correlations may relate specifically to the relationship between SOL muscle volume and $\dot{V} o_{2}$ peak. We observed correlations between SOL volume and $\dot{V} o_{2}$ peak (absolute and body mass normalized) in CHF, but no such correlation between $\dot{V} o_{2}$ peak and the MG or LG. Whilst similar correlations between the combined calf muscle volume and $\dot{V} \mathrm{O}_{2}$ peak have been observed (24), our finding reinforces the suggestion that the SOL muscle may be of particular importance as a determinant of functional capacity in CHF patients. This may relate to the SOL being an important determinant of aerobic potential due to its high oxidative capacity (13), as well as its key functional role in gait (25). Indeed the size of the gastrocnemius, a muscle known to have a lower oxidative capacity (13), did not correlate with $\dot{V} o_{2}$ peak in our study. It should be noted that similar to the present study, Harrington et al. (15) reported correlations between both quadriceps and thigh cross sectional area and absolute $\dot{V} o_{2}$ peak in CHF. However, the strong correlations observed in this study were likely due, in part, to the high covariance between body mass and muscle size and absolute $\dot{V} o_{2}$ peak. When thigh and quadriceps cross sectional area were correlated to body-mass normalized $\dot{V} o_{2}$ peak, relatively weak correlations were observed compared to that of the SOL in the present study. Furthermore, it should be noted that Harrington et al. (15) assessed quadriceps anatomical cross sectional area from a single axial plane image, and thus may not represent the average physiological cross sectional area of the muscle 
(typically requiring volume and fiber length and pennation angle measurements). Interestingly, our results also suggest that it may be reduced muscle volume per se, more so than PCSA that is linked with reduced $\dot{V}_{\mathrm{O}_{2}}$ peak in CHF.

\section{Paragraph number 32}

The link between the SOL function and $\dot{V}_{2}$ peak in CHF is also supported by the strong correlation between plantarflexor torque, for which the SOL is the major contributor, and $\dot{V} o_{2}$ peak in these patients. We are unaware of previous studies correlating plantarflexor strength and $\dot{V} o_{2}$ peak in CHF, although the strength of quadriceps has been previously shown to correlate with $\dot{V} o_{2}$ peak in CHF (26). That both the SOL muscle size and plantarflexor strength are correlated to $\dot{V} \mathrm{O}_{2}$ peak only in the CHF group supports the notion that peripheral determinants of $\dot{V} o_{2}$ peak may be present to a greater extent in CHF patients compared to healthy adults (8).

\section{Paragraph number 33}

Limitations. The present study compared CHF patients to control subjects matched for age and with similar adiposity. We cannot rule out the possibility that reduced activity levels in the CHF patients contributed, at least in part, to the reduced muscle and tendon size and the relationship between muscle volume, strength and exercise capacity observed in this study. There are several factors, however, that lead us to conclude that any influence of activity levels on our findings is likely to have been minimal. Firstly, all CHF patients were engaged in an exercise rehabilitation program and were undertaking supervised exercise 2-3 times weekly, similar to the activity of the control participants. Secondly, our finding that the reduction in muscle volume and PCSA as well as the relationship between muscle volume and $\dot{V} o_{2}$ peak is highly muscle specific would not be 
expected as a result of disuse alone, which should logically be expressed generically. For example, while disuse studies have found muscle loss in distal leg muscles (18), they also reported loss in the total lower limb lean mass (18). In contrast the present study only found a clear loss of muscle in the SOL, with no differences in total lower limb lean mass from DXA. Thirdly, the SOL has been reported to have a smaller reduction in size (or no reduction) compared to the MG and LG as a result of ageing/disuse $(3,30)$, whereas in the present study on CHF the SOL presents the largest reduction in size.

\section{Paragraph number 34}

Conclusion. The SOL is a key muscle involved in postural control and locomotion. It is classically considered to possess a higher proportion of type I muscle fibers than other muscles of the triceps surae and lower limb. Our findings indicate that SOL wasting is particularly marked in CHF, even when compared to other lower limb muscles in these individuals. Furthermore, the SOL volume correlated strongly with exercise capacity, whereas other individual leg muscle volumes did not, and is largely responsible for the correlation between plantarflexor strength and $\dot{V} o_{2}$ peak . For these reasons we propose that the SOL is a key muscle reflecting loss of function and exercise capacity in CHF, and may thus be a sentinel skeletal muscle in CHF patients. Finally, our results offer an evidence-base for including calf muscle-specific exercise training to help restore functional capacity in CHF.

\section{ACKNOWLEDGEMENTS}

The authors would like to acknowledge Mr. Tony Roby for building the water bath used for 3DUS measurements, Mr. Jack Liddell and Tim Verbeek for their help during data collection, and all of the participants who volunteered for this study. 


\section{CONFLICT OF INTEREST}

None of the authors involved in the present study have any conflict of interest, financial, personal or otherwise which would influence this research and the results do not constitute endorsement by ACSM.

\section{GRANTS}

This work was supported by a Grant-in-Aid (G09P 4469) from the National Heart Foundation of Australia to J.R., D.J.G., A.J.M. and D.G.L., and a thesis dissertation grant from the International Society of Biomechanics to F.A.P.

\section{LIST OF SUPPLEMENTAL DIGITAL CONTENT}

- Supplemental Digital Content 1. Text that describes the full details of the MRI procedures.

- Supplemental Digital Content 2. Text that describes the triceps surae tendon length estimates. 


\section{REFERENCES}

1. Arnold EM, Ward SR, Lieber RL, Delp SL. A model of the lower limb for analysis of human movement. Annal Biomed Eng. 2010; 38(2): 269-79.

2. Barber L, Barrett R, Lichtwark G. Validation of a freehand 3D ultrasound system for morphological measures of the medial gastrocnemius muscle. J Biomech 2009; 42(9): 1313-19.

3. Barber LA, Barrett RS, Gillett JG, Cresswell AG, Lichtwark GA. Neuromechanical properties of the triceps surae in young and older adults. Exp Gerontol. 2013;48(11): 114753.

4. Baxter JR, Piazza SJ. Plantarflexor moment arm and muscle volume predict torquegenerating capacity in young men. J Appl Physiol. 2013 in press

5. Besier TF, Sturnieks DL, Alderson JA \& Lloyd DG. Repeatability of gait data using a functional hip joint centre and a mean helical knee axis. J Biomech. 2003; 36(8): 1159-68.

6. Bland JM, Altman DG. Statistical methods for assessing agreement between two methods of clinical measurement. Lancet. 1986; 1(8476): 307-10.

7. Cicoira M, Zanolla L, Franceschini L, Rossi A, Golia G, Zamboni M, Tosoni P, Zardini P. Skeletal muscle mass independently predicts peak oxygen consumption and ventilatory response during exercise in noncachectic patients with chronic heart failure. J Am Coll Cardiol. 2001; 37(8):2080-85. 
8. Clark AL, Coats AJ. Exercise limitation in chronic heart failure: central role of the periphery. J Am Coll Cardiol. 1996; 28(5):1092-102.

9. Cohn JN, Johnson GR, Shabetai R, Loeb H, Tristani F, Rector T, Smith R, Fletcher R. Ejection fraction, peak exercise oxygen consumption, cardiothoracic ratio, ventricular arrhythmias, and plasma norepinephrine as determinants of prognosis in heart failure. Circulation. 1993; 87(6S): 15-16.

10. Delp MD, Duan C, Mattson JP, Musch TI. Changes in skeletal muscle biochemistry and histology relative to fiber type in rats with heart failure. J Appl Physiol. 1997; 83(4): 129199.

11. DeVita P, Hortobagyi T. Age causes a redistribution of joint torques and powers during gait. J Appl Physiol. 2000; 88(5): 1804-11.

12. Fülster S, Tacke M, Sandek A, Ebner N, Tschöpe C, Doehner W, Anker SD, von Haehling S. Muscle wasting in patients with chronic heart failure: results from the studies investigating comorbidities aggravating heart failure (SICA-HF). Eur Heart J. 2013; 34(7): 512-19.

13. Gollnick PD, Sjödin B, Karlsson J, Jansson E, Saltin B. Human soleus muscle: comparison of fiber composition and enzyme activities with other leg muscles. Pflugers Arch. 1974; 348: 247-255. 
14. Harridge SD, Magnusson G, Gordon A. Skeletal muscle contractile characteristics and fatigue resistance in patients with chronic heart failure. Eur Heart J. 1996; 17(6): 896-901.

15. Harrington D, Anker SD, Peng Chua T, Webb-Peploe KM, Ponikowski PP, Poole-Wilson PA, Coats AJS. Skeletal muscle function and its relation to exercise tolerance in chronic heart failure. J Am Coll Card. 1997; 30(7): 1758-64.

16. Héroux ME, Dakin CJ, Luu BL, Inglis JT, Blouin JS. Absence of lateral gastrocnemius activity and differential motor unit behavior in soleus and medial gastrocnemius during standing balance. J Appl Physiol. 2014; 116: 140-48.

17. Josephson RK. Dissecting muscle power output. J Exp Biol. 1999; 202: 3369-75.

18. Leblanc A, Schneider V, Evans H, Pientok C, Rowe R, Spector E. Regional changes in muscle mass following 17 weeks of bed rest. J Appl Physiol. 1992; 73(5): 2172-78.

19. Levine JA, Abboud L, Barry M, Reed JE, Sheedy PF, Jensen MD. Measuring leg muscle and fat mass in humans: comparison of CT and dual-energy X-ray absorptiometry. $J$ Appl Physiol. 2000; 88(2): 452-56.

20. Lichtwark GA, Wilson AM. Is Achilles tendon compliance optimised for maximum muscle efficiency during locomotion? J Biomech. 2007; 40(8):1768-75.

21. Lieber RL, Fridén J. Functional and clinical significance of skeletal muscle architecture. Muscle Nerve. 2000; 23(11): 1647-66. 
22. Maiorana A, O'Driscoll G, Cheetham C, Collis J, Goodman C, Rankin S, Taylor R, Green D. Combined aerobic and resistance exercise training improves functional capacity and strength in chronic heart failure. J Appl Physiol. 2000; 88(5):1565-70.

23. Mancini DM, Coyle E, Coggan A, Beltz J, Ferraro N, Montain S, Wilson JR. Contribution of intrinsic skeletal muscle changes to 31P NMR skeletal muscle metabolic abnormalities in patients with chronic heart failure. Circulation. 1989; 80(5): 1338-46.

24. Mancini DM, Walter G, Reichek N, Lenkinski R, McCully KK, Mullen JL, Wilson JR. Contribution of skeletal muscle atrophy to exercise intolerance and altered muscle metabolism in heart failure. Circulation. 1992; 85(4): 1364-73.

25. McGowan CP, Kram R, Neptune RR. Modulation of leg muscle function in response to altered demand for body support and forward propulsion during walking. J Biomech. 2009; 42(7): 850-56.

26. Minotti JR, Christoph I, Oka R, Weiner MW, Wells L, and Massie BM. Impaired skeletal muscle function in patients with congestive heart failure. Relationship to systemic exercise performance. J Clin Invest. 1991; 88(6): 2077-82.

27. Mitchell WK, Williams J, Atherton P, Larvin M, Lund J, Narici M. Sarcopenia, dynapenia, and the impact of advancing age on human skeletal muscle size and strength; a quantitative review. Front Physiol. 2012; 3: 260.

28. Moesteller RD. Simplified calculation of the body-surface area. N Engl J Med. 1987; 317: 1098. 
29. Moreira JBN, Bechara LRG, Bozi LHM, Jannig PR, Monteiro AWA, Dourado PM, Wisløff U, Brum PC. High-versus moderate-intensity aerobic exercise training effects on skeletal muscle of infarcted rats, J Appl Physiol. 2013; 114(8): 1029-41.

30. Morse CI, Thom JM, Birch KM, Narici MV. Changes in triceps surae muscle architecture with sarcopenia. Acta Physiol Scand. 2005; 183(3): 291-98.

31. Musch TI, Terrell JA. Skeletal muscle blood flow abnormalities in rats with a chronic myocardial infarction: rest and exercise, Am J Physiol. 1992; 262(2): 411-19.

32. Narici MV, de Boer MD. Disuse of the musculo-skeletal system in space and on earth. Eur J Appl Physiol. 2011; 111(3): 403-20.

33. Neptune RR, Kautz S, Zajac FE. Contributions of the individual ankle plantar flexors to support, forward progression and swing initiation during walking. J Biomech. 2001; 34(11):1387-98.

34. Panizzolo FA, Green DJ, Lloyd DG, Maiorana AJ, Rubenson J. Soleus fascicle length changes are conserved between young and old adults at their preferred walking speed. Gait Posture 2013; 38(4): 764-69.

35. Rubenson J, Henry HT, Dimoulas PM, Marsh RL. The cost of running uphill: Linking organismal and muscle energy use in guinea fowl (Numida meleagris). J Exp Bio. 2006; 209(13): 2395-408. 
36. Rubenson J, Pires JN, Loi HO, Pinniger GJ, Shannon DG. On the ascent: the soleus muscle is conserved to the ascending limb of the force length curve across gait mechanics in humans. J Exp Biol. 2012; 215(20):3539-51.

37. Smith DW, Gardiner B, Wang T, Lin Z, Day RE, Landao E, Rubenson J, Kirk TB, Lloyd DG, Wang A, Hardisty G, Zheng MH. A conceptual framework for computational models of Achilles tendon homeostasis. Wiley Interdisciplinary Reviews-Systems Biology and Medicine. 2013; 5(5): 523-38.

38. Stenroth L, Peltonen J, Cronin N, Sipilä S, Finni T. Age-related differences in Achilles tendon properties and triceps surae muscle architecture in vivo. J Appl Physiol. 2012; 113(10): 1537-44.

39. Toth MJ, Shaw AO, Miller MS, VanBuren P, LeWinter MM, Maughan DW, Ades PA. Reduced knee extensor function in heart failure is not explained by inactivity. Int J Card. 2010; 143(3): 276-82.

40. Williams AD, Selig S, Hare DL, Hayes A, Krum H, Patterson J, Geerling RH, Toia D, Carey MF. Reduced exercise tolerance in CHF may be related to factors other than impaired skeletal muscle oxidative capacity, J Card Fail. 2004; 10(2): 141-48 


\section{Figure captions}

Fig. 1. Schematic illustration of the procedure for obtaining triceps surae tendon length. (a) Subject marker set used for motion capture of skeletal elements, (b) Subject-specific scaled OpenSim model depicting the MG and SOL muscle tendon units, (c) Ultrasound measurement of muscle fascicle length and pennation angle, (d) Prediction of tendon length (see Text, Supplemental Digital Content 2, which describes the tendon length estimate).

Fig. 2. Muscle volume and physiological cross-sectional area (PCSA) values for the total triceps surae and individual plantarflexors. (a) Absolute volume, (b) lean body mass-normalized volume, (c) Absolute PCSA, and (d) BSA normalized PCSA. Data are means \pm S.D. The (\#) indicates a main effect ( $\mathrm{p}<0.05$; ANOVA) of group (CHF/Control; volume and PCSA collapsed over individual triceps surae muscles). The $(*)$ indicates a statistical difference (one-way ANOVA) between group (CHF/Control). A one-way ANOVA was performed only when an interaction effect between group and muscle were present. TS = triceps surae, $\mathrm{SOL}=$ soleus, $\mathrm{MG}=$ medial gastrocnemius, $\mathrm{LG}=$ lateral gastrocnemius, $\mathrm{CHF}=$ chronic heart failure). 
Fig.3. 3D-ultrasound and MRI-derived muscle volume. (a) Example of 3D volume rendering of the triceps surae created with 3D ultrasound (3DUS) and with MRI from the same individual. $\mathrm{SOL}=$ soleus, $\mathrm{MG}=$ medial gastrocnemius, $\mathrm{LG}=$ lateral gastrocnemius. $\mathbf{( b )}$ The correlation between volume calculation using 3DUS imaging and MRI (equation $y=0.998 x+9.365$, $r$ $=0.988, \mathrm{p}<0.001)$. The solid line represents the line of best fit and the dotted lines represent the $95 \%$ prediction interval. (c) Bland-Altman plot of the difference between 3DUS and MRI vs. the average of the MRI and 3DUS values. The horizontal lines on the plot represent the mean difference between MRI and 3DUS and the upper and lower 95\% limits of agreement.

Fig.4. Relationship between $\dot{V} o_{2}$ peak and leg muscle size. Relationship between absolute $\dot{V} o_{2}$ peak (ml $\mathrm{min}^{-1}$ ) and (a) leg lean mass (kg) and (b) triceps surae volume (ml). Relationship between body mass normalized $\dot{V} o_{2}$ peak and individual muscle volume in (c) the combined triceps surae, (d) soleus, (e) medial gastrocnemius, and (f) lateral gastrocnemius. Chronic heart failure subjects are displayed in grey triangles $(\Delta)$ and control subjects in open circles $(O)$.

Fig.5. Peak plantarflexor torque values and their relationship with $\dot{V} \mathrm{o}_{2}$ peak. Peak plantarflexor torque normalized to (a) body mass and (b) triceps surae PCSA. Data are means \pm S.D. (c) Relationship between body mass-normalized $\dot{V} o_{2}$ peak and body mass-normalized peak plantarflexor torque. Note one additional subject was added to the CHF group that was tested in a parallel study. Chronic heart failure subjects are displayed in grey triangles $(\Delta)$, and control subjects in open circles $(\bigcirc)$. 